\title{
Doppler Simulation and Analysis of SCME Channel Model
}

\author{
Xiaoyan Huang ${ }^{\mathrm{a},{ }^{* 1}}$, Xinhong Wang ${ }^{\mathrm{a},{ }^{* 2}}$, Yi Zhou ${ }^{\mathrm{a}}$, Ping Wang ${ }^{\mathrm{a}}$, Fuqiang Liu ${ }^{\mathrm{a}}$ \\ ${ }^{a}$ School of Electronics and Information Engineering, Tongji University, Shanghai, China
}

\begin{abstract}
In this contribution, the Doppler spectral properties are simulated and analyzed based on 3GPP Spatial Channel Model extension (SCME). SCME standard channel model is applied to the system evaluation and optimization in LTE and other future communication networks. Since future mobile communication systems demand for high moving speed, the simulation and analysis of Doppler characteristics become so significant. Therefore, we focus on the simulation of Doppler spectral properties in different scenarios of SCME channel model. The simulated Doppler power spectrum and Doppler delay spectrum are applicable to characterize Doppler properties of time-varying channels.
\end{abstract}

Index Terms: Time-varying Channel; Doppler Properties; Power Spectrum

(C) 2012 Published by MECS Publisher. Selection and/or peer review under responsibility of the Research Association of Modern Education and Computer Science

\section{Introduction}

An accurate knowledge of modeling of propagation channels is a prerequisite for the design and performance assessment of advanced wireless communication systems. Since 1998, ETSI's standardization of 3rd-generation mobile systems has been carried out in the 3rd Generation Partnership Project (3GPP). In 2003, 3GPP proposed SCM (Spatial Channel Model) [1] which gives detailed description of the link level and system level spatial model for CDMA system. The carrier frequency is $2 \mathrm{GHz}$ and the bandwidth is $5 \mathrm{MHz}$. The European WINNER (Wireless World Initiative New Radio) project was launched in 2004 [2] and the 3GPP SCM for a MIMO wireless system was adopted to simulate the wideband fast fading scenarios.

However, channel model supporting $20 \mathrm{MHz}$ is needed for 3GPP LTE (Long Term Evolution) purposes. The first extension of the 3GPP/3GPP2 model was proposed in 2005. It was named SCME (SCM-Extension) channel model [3]. This spatial channel model broadened the channel bandwidth from $5 \mathrm{MHz}$ to $100 \mathrm{MHz}$ and covered frequency up to $5 \mathrm{GHz}$. Another purpose of the extension is to keep the model simplicity and backward compatibility with SCM.

Recently more and more researches emphasize on the characteristics of time-varying channels in mobile scenarios. Doppler spectral properties are used to describe the time-varying characteristics of propagation paths.

Supported by National Natural Science Foundation of China (61073153)

* Corresponding author:

E-mail address: ${ }^{* 1}$ hbswallow88@163.com; ${ }^{* 2}$ wang xinhong@163.com 
For example, the Doppler spectrum for stationary transceivers with randomly distributed moving scatterers around was analyzed in [4]. It has been known that Doppler spectra are sharply peaked at zero frequency. However, the realistic measured distribution depends on the moving scatterers and the estimation accuracy. In [5] the spectral properties of signal power variations in the frequency domain were described. It was also illustrated that the type of fast fading can be estimated from the distribution and the magnitudes of K-factor within the Doppler bandwidth.

To estimate the parameters of the propagation paths, high resolution algorithms such as SAGE (SpaceAlternating Generalized Expectation maximization) [6] and ESPRIT (Estimation of Signal Parameters via Rotational Invariance Technique) [7] can be used. To reduce the computational costs in the simulation of highquality channel waveforms, a new parameter computation method was proposed in [8]. It can be applied on any given asymmetrical Doppler power spectrum. Also some researches focused on the channel sounding and measurement like in relay channels [9].

A few researches focus on the comparison of the performance of the standard channel models. In [10] different features and performances of the SCM, SCME and WINNER channel models were described in terms of the modeling methodology and features such as applicability range, system level description and complexity issues. The performance figures of temporal auto-correlation functions and frequency correlation functions were also given. Nevertheless, Doppler characteristics in SCME channel model have not been involved. Thus we focus on the simulation and analysis of Doppler spectral properties.

In this contribution, we characterize the time-variability of SCME channel model. The mobile status considered here covers mostly encountered situations in non-static environment typical for cellular environment. Doppler power spectrum and Doppler delay spectrum in the three scenarios are illustrated. The simulation results can be applied to model the Doppler characteristics in time-varying scenarios in mobile communication systems.

The paper is organized as follows. The theoretical background of Doppler spectral properties in SCME channel model is presented in Section II. Doppler simulation and analysis of Doppler power spectrum and Doppler delay spectrum are given in Section III. Finally, conclusive remarks are made in Section IV.

\section{The Doppler Spectral Properties in SCME Channel Model}

The classical tub-shaped Doppler spectrum was derived based on the Jakes' model [11] under the assumption that the transmitter as well as the scatterer is stationary and the receiver is moving. In such a case, the Doppler frequency of a propagation path is a function of the receiver's speed and the angle between the receiver's velocity and the line of sight between the transmitter and the receiver. This method is adopted in the 3GPP SCME standard channel models to compute the Doppler frequency of individual paths.

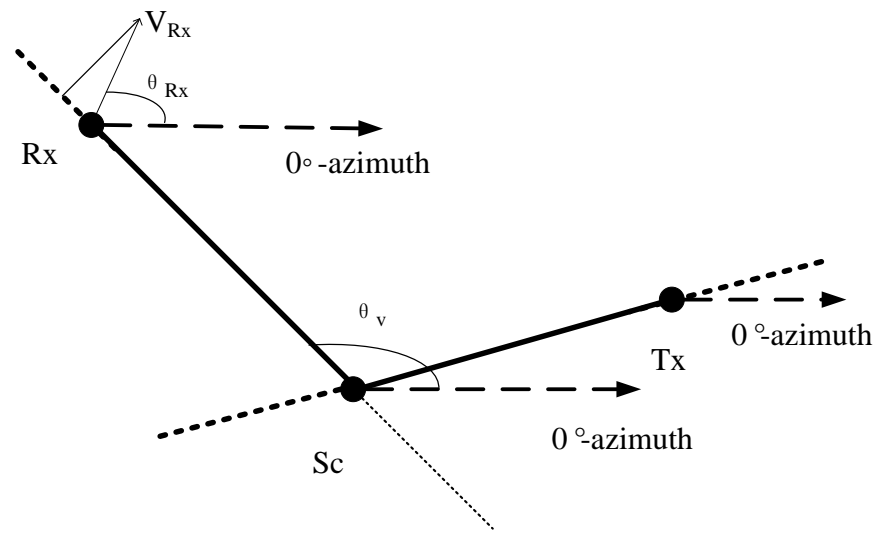

Fig. 1. The schematic of moving Rx, stationary Tx and scatterer 
Fig. 1 depicts a diagram of the considered scenario where $\theta_{\mathrm{R}_{\mathrm{X}}}$ denotes the azimuth of travel of the receiver, $\mathrm{v}_{\mathrm{MS}}$ denotes the moving speed of the receiver, $\theta_{\mathrm{v}}$ denotes the azimuth of arrival of the $l$ th path.

The linear time-variant channel impulse response $h(t, \tau)$ describes the relation of an input signal $x(t)$ to an output signal $\mathrm{y}(\mathrm{t})$

$$
y(t)=\int_{-\infty}^{+\infty} x(t-\tau) \cdot h(t, \tau) d \tau+n(t)
$$

$h(t, \tau)$ is dependent on two time variables, where ${ }^{t}$ is the overall time and $\tau$ the time delay [12]. $n(t)$ includes noise and interfering signals. The most important channel function for modeling is the time-variant frequency response (FR) $T(t, f)$ which can be converted to $h(t, \tau)$ by

$$
h(t, \tau)=\int_{-\infty}^{+\infty} T(t, f) e^{j 2 \pi f \tau} d f
$$

We first derive the autocorrelation function $R(\Delta t)$ to find the spectrum

$$
\begin{aligned}
\mathrm{R}(\Delta \mathrm{t} ; \tau) & =\frac{1}{2} \mathrm{E}\left\{\mathrm{h}(\mathrm{t}+\Delta \mathrm{t}) \mathrm{h}^{*}(\mathrm{t})\right\} \\
& =2 \delta_{0}^{2} \mathrm{~J}_{0}(2 \pi \Delta)
\end{aligned}
$$

where $\delta_{0}^{2}$ denotes the signal variance, $\mathrm{J}_{0}(2 \pi \Delta)$ denotes the Bessel function of 0 -th order.

$$
\mathrm{J}_{0}(\mathrm{z})=\frac{2}{\pi} \int_{0}^{\frac{\pi}{2}} \cos (\mathrm{z} \sin \mathrm{a}) \mathrm{da}
$$

The spectrum is the Fourier transform of the autocorrelation function

$$
\mathrm{S}_{\mathrm{C}}\left(\mathrm{f}_{\mathrm{d}} ; \tau\right)=\int_{-\infty}^{+\infty} \mathrm{R}_{\mathrm{C}}(\Delta \mathrm{t} ; \tau) \mathrm{e}^{-\mathrm{j} 2 \pi \mathrm{f}_{\mathrm{d}} \Delta \mathrm{t}} \mathrm{d} \Delta \mathrm{t}
$$

In SCME channel model, signals arriving at the moving receiver come from different paths. The moving velocity will result in different Doppler shift of each multipath component. Thus different Doppler power spectrum properties are obtained. It is assumed that the angle of arrivals follows a uniform distribution. The Doppler power spectrum has the symmetric Jakes power spectrum density function [13].

$$
\mathrm{S}_{\mathrm{C}}\left(\mathrm{f}_{\mathrm{d}} ; \tau\right)= \begin{cases}\frac{\delta_{\mathrm{u}}^{2}}{\pi \mathrm{f}_{\max } \sqrt{1-\left(\mathrm{f} / \mathrm{f}_{\max }\right)^{2}},}, & |\mathrm{f}|<\mathrm{f}_{\text {max }} \\ 0, & |\mathrm{f}| \geq \mathrm{f}_{\text {max }}\end{cases}
$$

where $S_{C}\left(f_{d}\right)$ is the Doppler power spectrum, and $f_{d}$ is the Doppler frequency.

The classic Doppler equation is given by:

$$
\mathrm{f}_{\mathrm{d}}=\lambda^{-1} 2 \mathrm{v} \cos \theta
$$

where $f_{d}$ usually corresponds to the energy peak of the Doppler spectrum. $\lambda, v$ and $\theta$ are the wavelength, the velocity and the Doppler angle, respectively.

A most sifnificant aspect of channel modeling is the proper representation of the channel fading behavior of $h(\tau ; t)$ or $h(k)$ when the statistical behavior of the physical channel $c(s ; t)$ is known from measurements or 
widely accepted specifications. Short-term channel variations are characterized by the WSSUS (Wide Sense Stationary Un-correlated Scattering) scattering function [14]

$$
S\left(f_{d} ; \tau\right)=\sum_{n=1}^{N} S_{n}\left(f_{d}\right) \cdot \sigma\left(\tau-\tau_{n}\right)
$$

where $\tau_{m}$ is the biggest differential delay. The random delays of the paths have been seen experimentally to follow an approximate exponential distribution. Thus, they can be expressed as

$$
\tau_{\mathrm{n}}^{\prime}=-\mathrm{r}_{\mathrm{DS}} \sigma_{\mathrm{DS}} \ln \mathrm{z}_{\mathrm{n}}, \mathrm{n}=1, \ldots, \mathrm{N}
$$

where $z_{n}(n=1, \ldots, N)$ are independent identically distributed (i.i.d.) random variables with uniform distribution $\mathrm{U}(0,1), \mathrm{r}_{\mathrm{DS}}=\sigma_{\text {delays }} / \sigma_{\mathrm{DS}}$ signifies the ratio of the two time constants and $\sigma_{\mathrm{DS}}$ is related to the power density as a function of delay. For simplicity, $\mathrm{r}_{\mathrm{DS}}$ is chosen to be a constant, independent of the particular realization of $\sigma_{\mathrm{DS}}$.

The average powers of the $\mathrm{N}$ paths can be expressed as

$$
\mathrm{P}_{\mathrm{n}}^{\prime}=\mathrm{e}^{\frac{\left(1-\mathrm{r}_{\mathrm{DS}) \tau_{\mathrm{n}}}\right.}{\mathrm{r}_{\mathrm{DS}} \cdot \sigma_{\mathrm{DS}}}} \cdot 10^{-0.1 \varepsilon_{\mathrm{n}}}, \mathrm{n}=1, \ldots, \mathrm{N}
$$

where $\varepsilon_{\mathrm{n}}$ for $\mathrm{n}=1, \ldots, \mathrm{N}$ are i.i.d. Gaussian random variables with standard deviation $\sigma_{\mathrm{RND}}=3 \mathrm{~dB}$, signifying the fluctuations of the powers away from the average exponential behavior.

In SCME channel model, both the transmitter and the scatterers are stationary and the receiver is moving in three scenarios (Urban Macro、Suburban Macro and Urban Micro). Thus the Doppler frequency of a propagation path induced by a stationary scatterer can be calculated as

$$
\mathrm{f}_{\mathrm{d}}=\mathrm{v}_{\mathrm{R}_{\mathrm{X}}} \cos \left(\theta_{\mathrm{v}}-\theta_{\mathrm{R}_{\mathrm{X}}}\right) * \mathrm{f}_{\mathrm{c}} / \mathrm{C}
$$

where $C$ is the light speed, $f_{c}$ is the center frequency, $v_{M S}$ is the moving speed of the mobile station, $\theta_{\mathrm{v}}$ represents the sub-paths in the dispersive path which follows the von-Mises distribution $\vartheta\left(\overline{\emptyset_{2}}, K_{\emptyset_{2}}\right)$, where $\bar{\emptyset}$ denotes the mean azimuth of arrival, and $\mathrm{K}_{\emptyset_{2}}$ denotes the concentration parameter.

\section{Doppler Simulation and Analys}

In SCME channel model, the 20 sub-paths are split into mid-paths and then moved to different delays, thus introduce in intra-cluster delay spread. The COST-Walfish-Ikegami-Model (COST-WI) model is proposed for all scenarios. Unlike the 3GPP SCM which only consider line of sight (LOS) in Urban Micro, LOS is extended to cover also Urban Macro and Suburban Macro. To keep the backward compatibility with SCM, the complexity increases, thus quasi-deterministic drifting of small-scale parameters inside drop is evaluated.

The scenarios with stationary transmitter and scatterer and moving receiver can be found in cellular networks, as well as in vehicular environments such as vehicle-to-infrastructure communications. In this section, simulations are performed to illustrate the Doppler characteristics of SCME channel model. In the three scenarios in SCME channel model, the simulation parameters are as follows. The environment is with only vertical polarization and the base station (BS) is equipped with a three-sector antenna. The angle between the broadside of the mobile station array and the absolute North reference direction $\Omega_{\mathrm{MS}}$ is assumed to be 83 . The default value of the mobile station velocity is $60 \mathrm{~km} / \mathrm{h}$. For Urban Macro and Suburban Macro scenarios, the distance from BS to BS is approximately $3 \mathrm{~km}$ and the BS antenna is above rooftop height. For Urban Micro scenario, the distance from BS to BS is less than $1 \mathrm{~km}$ and the BS antenna is at rooftop height. 


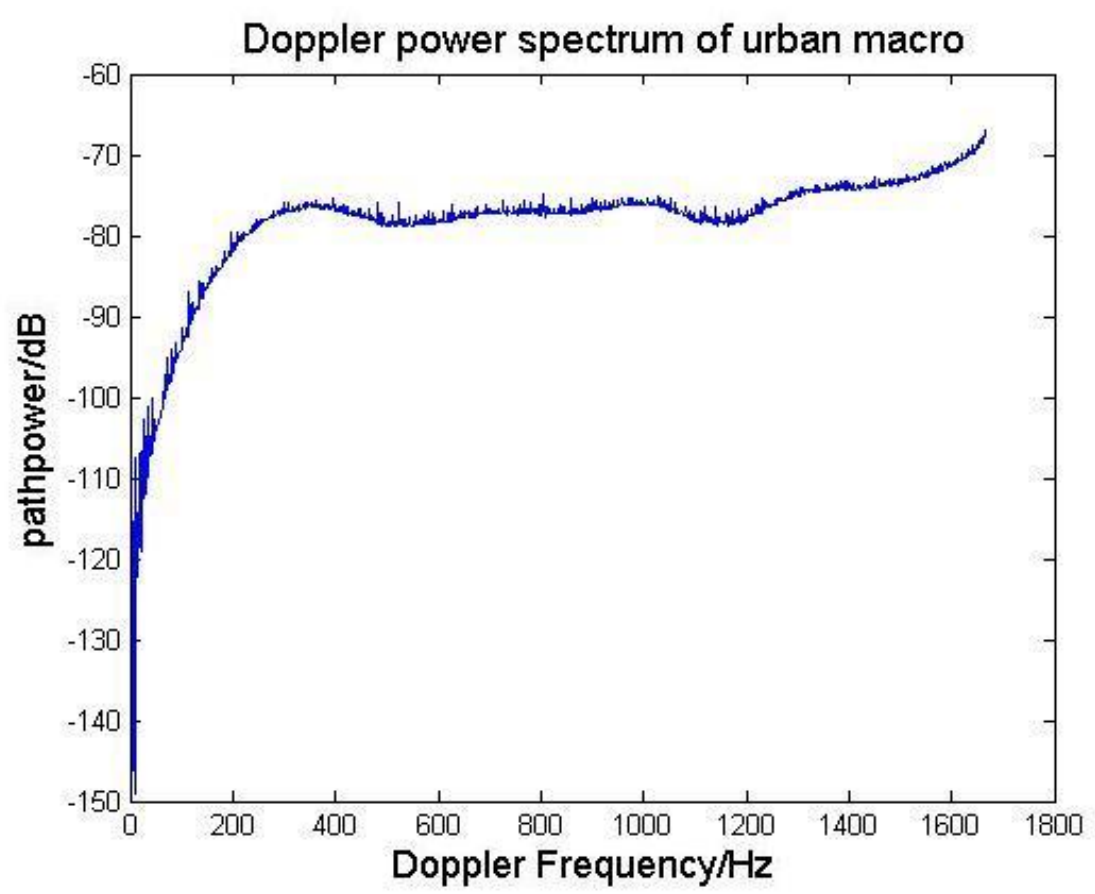

Fig.2(a) Doppler power spectrum of Urban Macro



Fig.2(b) Doppler power spectrum of Suburban Macro 


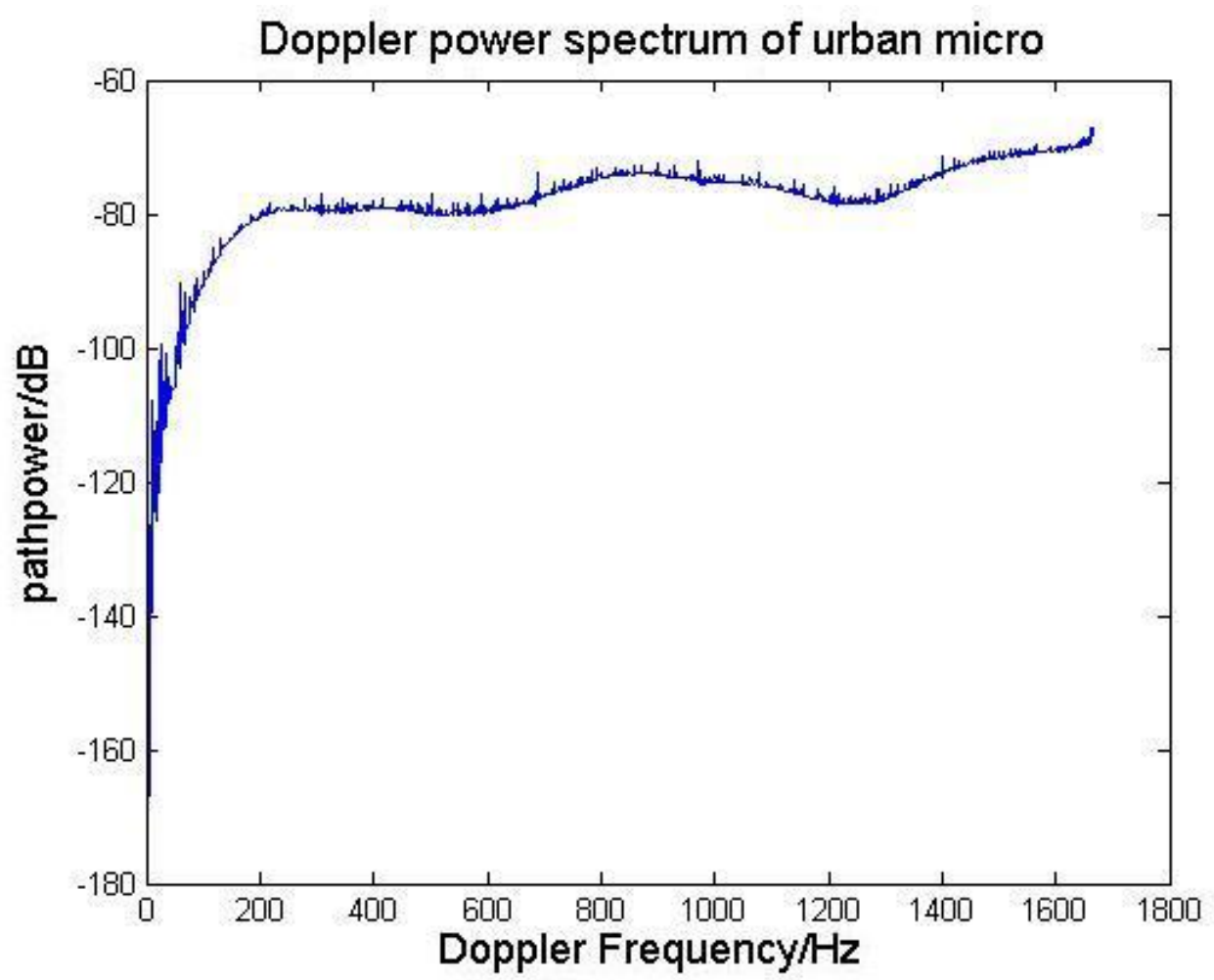

Fig.2(c) Doppler Power spectrum of Urban Micro

Fig. 2. Doppler power spectrum in SCME channel model

Fig. 2 (a) (b) (c) depict Doppler power spectrum of Urban Macro、Suburban Macro and Urban Micro scenarios in SCME channel model respectively. The spectra show very similar results in all compared scenarios. From Fig. 2 (a) and (c) for urban scenarios we can see that the power distribution in the Doppler frequencies range from $300 \mathrm{~Hz}$ to $1700 \mathrm{~Hz}$ is nearly the same. At the same time there are some differences in the Doppler frequencies range from $0 \mathrm{~Hz}$ to $300 \mathrm{~Hz}$. The scatterers in Urban Macro scenario are sparser than in Urban Micro scenario. The power is quite different near the zero Doppler frequency. The spectrum is somewhat different in Fig. 2 (b). The scatterers such as buildings and trees spread randomly in Suburban Macro scenario and thus show some fluctuations.

Fig. 3 (a) (b) (c) depict Doppler delay spectrum of Urban Macro、Suburban Macro and Urban Micro scenarios in SCME channel model respectively. Due to the introduction of intra-cluster delay spread, the delay spans a wide range. From Fig. 3 (a) and (c) for urban scenarios we can see that the path delays span mostly

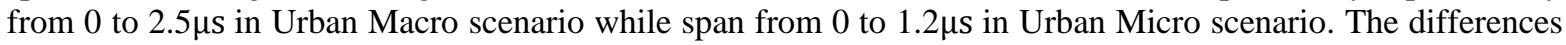
between them are on account of the distances between the base stations. For Fig. 3 (b), the delay in Suburban Macro scenario is smaller than in Urban Macro scenario. This is because the number of scatterers in suburban environment is much less than that in urban environment. As we can gain from Fig. 3 (a) and (c), the uniform Doppler Frequency distributions show that the scatterers are distributed universally and uniformly in urban scenario. The spectrum is somewhat different in Fig. 3 (b). It's a little dense in the frequency range from $1200 \mathrm{~Hz}$ to $1700 \mathrm{~Hz}$. This is due to the scatterers in suburban environment are distributed not so regularly. This result is in accord with the results we get form the continuous Doppler power profiles. 


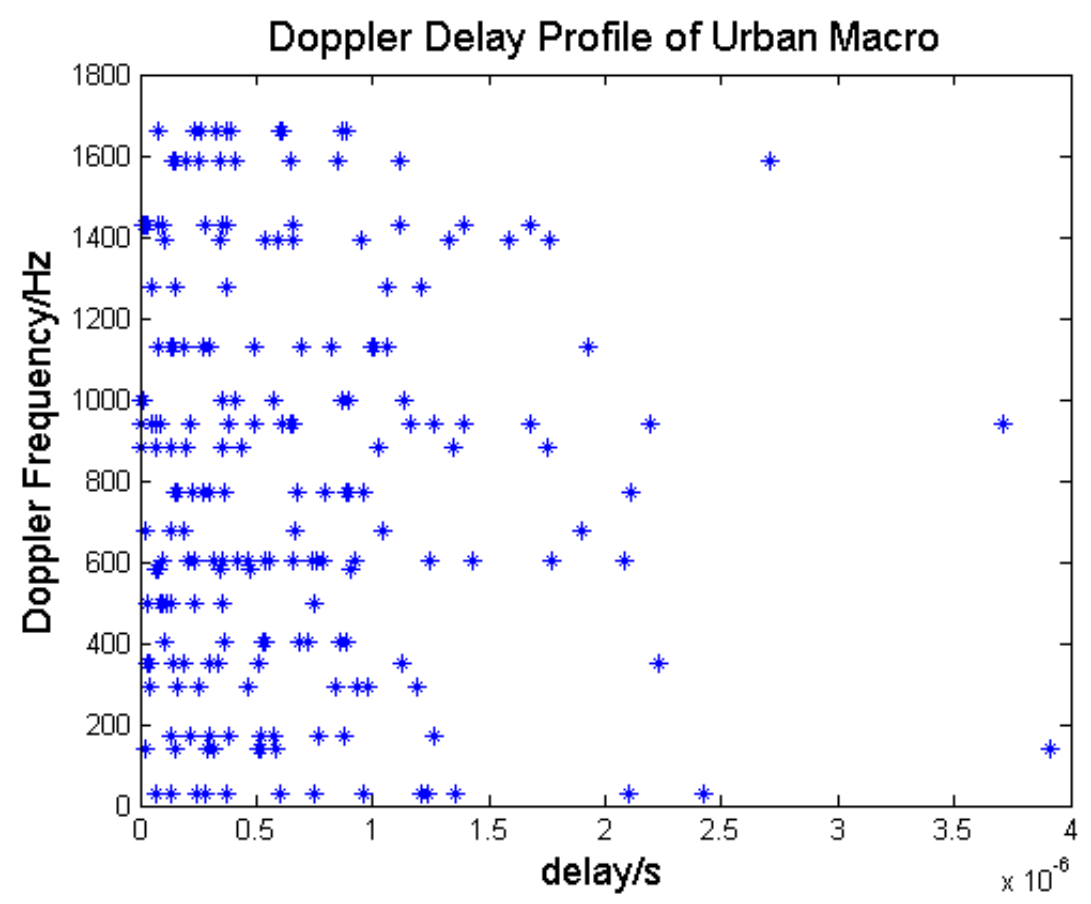

Fig.3(a) Doppler Delay spectrum of Urban Macro



Fig.3(b) Doppler Delay spectrum of Suburban Macro 


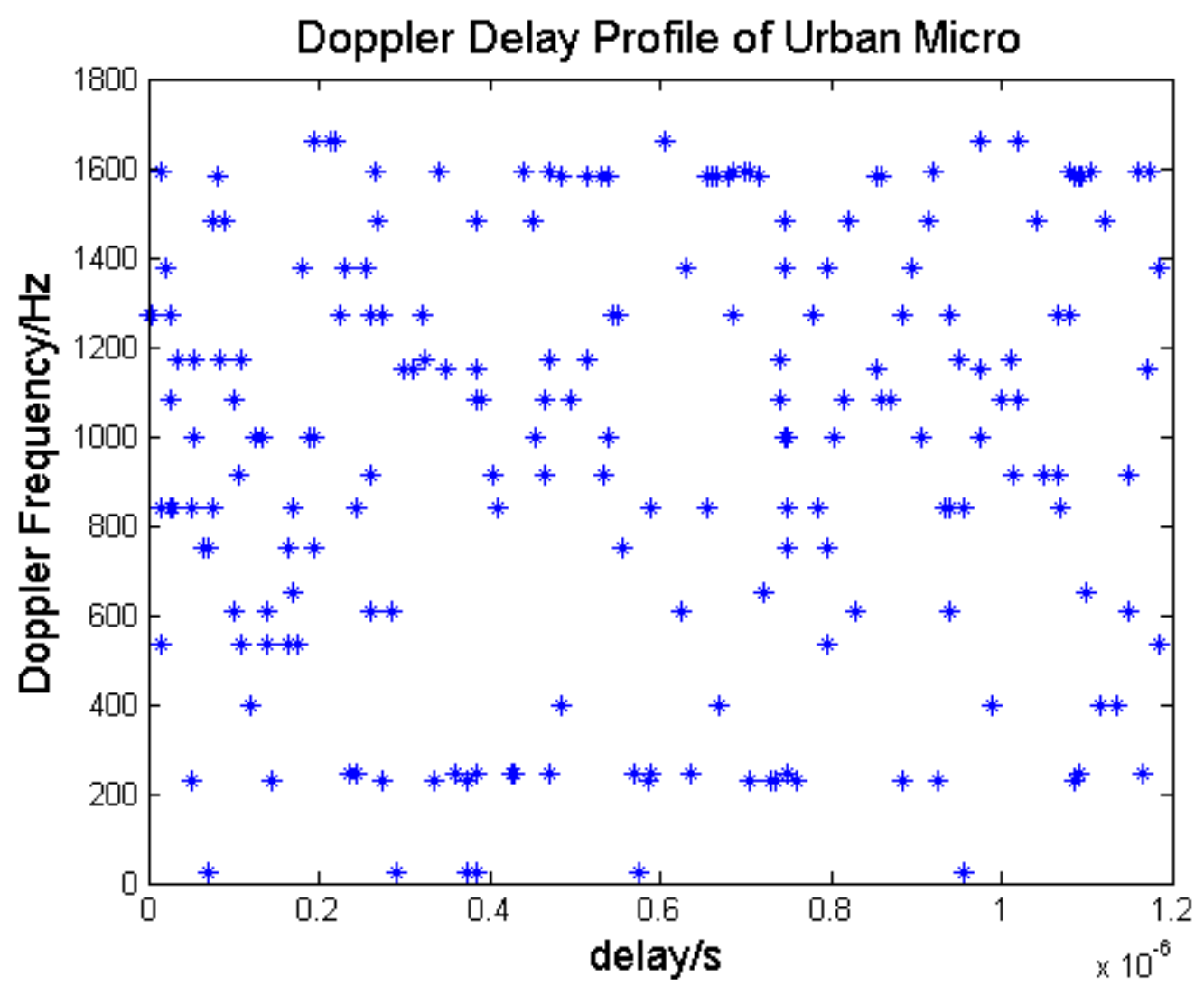

Fig.3(c) Doppler Delay spectrum of Urban Micro

Fig. 3. Doppler delay spectrum in SCME channel model

\section{Conclusion}

In the current work, we simulate the short-term time variation of SCME channel model. The considered scenarios in SCME channel model where the transmitter and the scatterers are stationary and the receiver is moving are the general cases in mobile communication systems. Doppler power spectrum and Doppler delay spectrum are simulated and analyzed. The simulation results are useful to describe the full characteristics of time-variant channels in mobile communication systems.

\section{References}

[1] 3rd Generation Partnership Project; "Tr 25.996 spatial channel model for multiple input multiple output (mimo) simulations, v6.1.0,” Tech. Rep., 2003-09.

[2] P. Kÿosti, J. Meinilä, L. Hentilä, X. Zhao, T. Jämsä, C. Schneider,M. Narandzić, M. Milojević, A. Hong J. Ylitalo, V.-M. Holappa,M. Alatossava, R. Bultitude, Y. de Jong and T. Rautiainen,"WINNER II Channel Models D1.1.2 V1.1,” no. IST-4-027756WINNER II, D1.1.2 V1.1, 112007. 
[3] D. S. Baum, J. Salo, G. Del Galdo, M. Milojevic, P. Kyösti, and J. Hansen, An Interim Channel Model for Beyond-3G Systems, in Proc. IEEE VTC 2005 Spring, Stockholm, May 2005

[4] Jørgen Bach Andersen, Jesper Ødum Nielsen, "Doppler Spectrum from Moving Scatterers in a Random Environment," IEEE Transactions on Wireless Communications, vol. 8, no. 6, pp. 3270, June 2009.

[5] Nathan Blaunstein, Dimitry Katz, and Masashi Hayakawa, "Spectral Properties of Modulated Signal in the DopplerDomain in Urban Radio Channels With Fading," IEEE Transactions on Antennas and Propagation, vol. 58, no. 8, pp. 2795-2800, August 2010.

[6] B. H. Fleury, M. Tschudin, R. Heddergott, D. Dahlhaus, and K. I.Pedersen, "Channel parameter estimation in mobile radio environmentsusing the sage algorithm," IEEE Journal on Selected Areas in Communications,vol. 17, no. 3, pp. 434-450, Mar. 1999.

[7] R. K. T. Paulraj, A.; Roy, "Estimation of signal parameters via rotational invariance techniques/sup 1/esprit." Nineteeth Asilomar Conference on Circuits, Systems and Computers, November 1985, pp. 83-89.

[8] Carlos A. Guti'errez-D'iaz-de-Le'on and Matthias P"atzold, "Efficient Sum-of-Sinusoids-Based Simulation of Mobile Fading Channels with Asymmetrical Doppler Power Spectra," 4th International Symposium on Wireless Communication Systems, 2007. ISWCS 2007, pp. 246-251, Dec. 2007.

[9] Ping Wang, Yi Zhou, Xuefeng Yin, Xiaoyan Huang, and Fuqiang Liu, "Measurement and Analysis of Relay Channels in Indoor Environments," 6th International Conference on Wireless Communications Networking and Mobile Computing (WiCOM), Sept. 2010.

[10] Narandzic, M.; Schneider, C.; Thoma, R ; Jamsa, T.; Kyosti, P.; Xiongwen Zhao, "Comparison of SCM, SCME, and WINNER Channel Models,” IEEE 65th Vehicular Technology Conference, 2007. VTC2007Spring.pp. 413-417, 2007.

[11] W. C. Jakes, Microwave Mobile Communications. New York: IEEE Press, 1974.

[12] T. S. Rappaport,Wireless Communications: Piscataway, NJ: IEEE Press,1996.1.

[13] T. S. Rappaport, Wireless Communications: Principles \& Practice, Upper SaddleRiver, NJ: Prentice Hall PTR, 1996.

[14] Parsons. The Mobile Radio Propagation Channel. Pentech Press: London, 1992. 